\title{
Die Tücken der traumatherapeutischen Praxis
}

\section{Ein Expertinnengespräch mit der Psychotraumatologin Michalea Huber, geführt von Johanna Schwetz-Würth}

\author{
Michaela Huber · Johanna Schwetz-Würth
}

Online publiziert: 10. Mai 2017

(C) Der/die Autor(en) 2017. Dieser Artikel ist eine Open-Access-Publikation.

Die Begleitung von Menschen mit komplexen Traumafolgestörungen im niedergelassenen sowie im stationären Bereich ist häufig mit großen Schwierigkeiten und Herausforderungen auf verschiedenen Ebenen verbunden. Im folgenden Gespräch werden einige Aspekte dazu diskutiert, insbesondere aber Frage herausgearbeitet, wie adäquate und langfristig passende Hilfe sowohl für Betroffene als auch die Behandelnden aussehen könnte?

Psychotherapeutlnnen wissen wenig über Traumafolgestörungen - mit gravierenden Folgen für die Betroffenen

JOHANNA SCHWETZ-WÜRTH: Liebe Michaela, ich möchte unser Gespräch unter den Titel: „Die Tücken der traumatherapeutischen Praxis“ stellen. In Deutschland wie auch in Österreich verfügen viele psychotherapeutischen KollegInnen kaum über traumatherapeutische Weiterbildung oder Expertise, behandeln aber sehr wohl - wissend oder nicht wissend - traumatisierte KlientInnen. Wie groß ist da der Prozentsatz in Deutschland?

MICHAELA HUBER: Es gibt erste Befragungen darüber, etwa von der Phönix-Initiative, die zeigen, dass die betroffenen Menschen mit Traumafolgestörungen sehr unglücklich darüber sind, dass die meisten PsychotherapeutInnen und PsychiaterInnen oder Ärz-

Diese Arbeit ist Teil des Leitthemas „Traumatherapie in der Praxis“.

\section{Huber $(\square)$}

Söseweg 26, 37081 Göttingen, Deutschland

huber_michaela@t-online.de

J. Schwetz-Würth

Schottenfeldgasse 15/3, 1070 Wien, Deutschland praxis@johanna-schwetz.at
tInnen, die sie aufgesucht haben, leider sehr wenig von Traumafolgestörungen gewusst haben. Daher seien sie entweder symptomorientiert auf Essstörungen, andere Suchterkrankungen, Depressionen, Angststörungen oder allgemein verhaltenstherapeutisch, tiefenpsychologisch oder nur medikamentös behandelt worden. Alles wenig hilfreich! Denn die spezifischen Bedingungen von traumatisierten Menschen sind ja, dass sie in körperliche und emotionale Zustände geraten, die sie mit ihrem Alltags-Ich nicht unter Kontrolle haben. Zahlenmäßig haben sich etwa $10 \%$ der Betroffenen einigermaßen traumatherapeutisch irgendwann, irgendwo verstanden gefühlt, 80-90\% waren immer wieder auf der Suche oder in der Warteschleife, und viele davon ziemlich unglücklich.

JOHANNA SCHWETZ-WÜRTH: Was würdest Du sagen, was sind die größten Schwierigkeiten, mit denen komplex traumatisierte KlientInnen konfrontiert sind, wenn sie an solche, nicht ausgebildeten KollegInnen geraten? Was ist das Schwierigste?

MICHAELA HUBER: Ich glaube, es gibt zwei grundlegende Schwierigkeiten. Die erste besteht darin, dass die Menschen in einer Traumasituation Unerträgliches ganz alleine durchstehen mussten, das sie nicht verdauen konnten, und dabei existentielle Einsamkeit erlebt haben, entfremdet von anderen Menschen. Das erfordert eine Zugangsweise von helfenden Personen, die speziell wissen, wie sich jemand fühlt, der diese Zustände von seelischer Erschütterung erlebt hat, wie fragmentiert die Person die Situation wahrgenommen und gespeichert hat und wie verzweifelt sie versucht, die Menschen und sich selbst zu verstehen und das Unverständliche auszusortieren. Das ist die eine große Schwierigkeit. Die andere ist, wie arbeitet man mit Wahrnehmungs-, Erinnerungs- und Körpermaterial, das Extremzustände wiederspiegelt, die 
dem Alltags-Ich entfremdet sind. Das sind zwei zentrale Probleme, also zusammengefasst: der Bindungsund Beziehungsaspekt und der Umgang mit diesen dissoziativen States, die sehr besonders sind. Hinzu kommt das, was Judith Herman so treffend ausgedrückt hat: „Bei der traumatischen Übertragung geht es immer um Leben und Tod!“ Das heißt, es gibt intensivste Übertragungs- und Gegenübertragungsphänomene, es gibt immer einhundert Prozent, immer ganz oder gar nicht, da oder weg, Flucht oder Kampf, Übererregung oder Unterwerfung. In diesen Dynamiken als PsychotherapeutIn oder PsychiaterIn eingebunden zu sein, ist extrem anstrengend und herausfordernd.

Extremzustände in der psychotherapeutischen Praxis - die Arbeit mit komplex traumatisierten KlientInnen

JOHANNA SCHWETZ-WÜRTH: Was würdest Du sagen, wenn wir von einer wohlmeinenden Therapeutin ausgehen, die aber leider noch keine Weiterbildung im Bereich Trauma gemacht hat, aber vielleicht dieses Gespräch liest, woran würde diese in der Praxis erkennen, dass sie höchst wahrscheinlich einer komplex traumatisierten Klientin, einem Klienten gegenübersitzt? Was wäre so ein erstes Kriterium, wo man denken müsste, dass da etwas anders ist?

MICHAELA HUBER: Ich würde sogar damit beginnen, zu fragen, wie erkennt die Therapeutin, der Therapeut eine AKUT-Traumatisierung? Viele komplex traumatisierten Menschen sind nach wie vor in Lebensumständen, die intensiv re-traumatisieren und so wie ein Akut-Trauma aussehen oder die nach wie vor Traumasituationen darstellen. Das heißt, wenn man jemandem gegenübersitzt, der starke Erregungszustände hat und auch - danach gefragt - von extremen Untererregungszuständen, in Form von depressiven Einbrüchen oder extremen Erschlaffungszuständen berichten kann, dann würde ich auf eine stressbedingte Störung hin untersuchen. Danach würde ich fragen, ob die Person sich derzeit in Lebenszuständen befindet, die extrem stressig sind. Die meisten Menschen sagen nicht, ich bin traumatisiert. Diejenigen, die laut mit einer Traumadiagnose herumlaufen, die muss man auch noch genauer untersuchen, ob das wirklich stimmt, weil es inzwischen viele gibt, die gerne diese Etiketten benutzen. Die Physiologie, also die Körpersymptomatik, ist letztlich immer das Entscheidende, denn das Implizite oder Traumagedächtnis ist vor allem somatopsychisch organisiert. Das heißt, ein Auslöser wie etwa ein harmloser Geruch oder Anblick löst extrem starke Körperreaktionen aus. Dazu kommt, dass Menschen in ihrer Identität, und das betrifft vor allem die komplex Traumatisierten, extrem gestört sind in folgender Hinsicht: diese Menschen haben ein extrem schlechtes Selbstwertgefühl, obwohl sie durchaus viele Kompetenzen haben. Sie haben zahlreiche Bindungs- und Beziehungsabbrüche in ihrer Biographie und sind darüber sehr verzweifelt, sie haben Körperzustände, die sie nicht unter Kontrolle haben, sie können Impulse und Emotionen sehr schlecht regulieren, obwohl sie es verzweifelt versuchen und sie haben Probleme damit, einen Sinn in ihrem Leben zu finden und brechen immer wieder zusammen bis hin zu suizidalen Krisen oder schweren Selbstschädigungen oder Selbstverletzungen. Das kennzeichnet komplex traumatisierte Menschen.

JOHANNA SCHWETZ-WÜRTH: Wenn die therapeutische Beziehung zwischen den beiden passt, was sollte die Therapeutin tun, wie sollte sie weiter vorgehen, um dieser Klientin optimal zu helfen?

MICHAELA HUBER: Das kommt darauf an, ob die therapeutische Beziehung zu diesem Zeitpunkt gut ist bzw. ob die Klientin existentiell von der TherapeutIn abhängig ist. Die therapeutische Person, die eine gute Beziehung hat, sollte immer versuchen, sich sofort kompetente und engagierte Fachpersonen an die Seite zu holen, sie sollte die Beziehung zu dieser Klientin halten und sich intensiv weiterbilden und in Supervision begeben. Wenn die Beziehung noch nicht stimmt und die Klientin nicht existentiell abhängig ist, sollte man sie an eine versierte Kollegin weiter verweisen. Da gibt es das bekannte Problem, dass die meisten KollegInnen, die Trauma-Kenntnisse und Erfahrungen haben, überlaufen sind. Das heißt, ich würde immer dazu raten, halten Sie den Kontakt zu der Klientin, einfach freundlich unterstützend, seien Sie der Fels in der Brandung und holen Sie sich sofort fachliche Unterstützung an ihre Seite, bauen Sie ein kleines Helfernetzwerk auf, damit die Klientin nicht fallen gelassen wird.

\section{Erfahrene und versierte TraumatherapeutInnen - überfordert und wenig anerkannt}

JOHANNA SCHWETZ-WÜRTH: Gehen wir zu den KollegInnen, die sich spezifisch weitergebildet und qualifiziert haben und auch in diesem Feld erfahren sind. Wie siehst Du das in Deutschland, wie hoch ist die Anerkennung dieser Weiterbildungen bzw. Zertifizierungen im deutschen Gesundheitssystem? Wird das bereits als wichtige Kompetenz und Qualifikation wahrgenommen?

MICHAELA HUBER: Das kommt darauf an. Wenn die Leitung der Institution grundsätzlich der Meinung ist, dass das alles Quatsch ist, dass die klassisch symptomorientierte Behandlung ausreichend ist, dann wird man diese KollegInnen nicht anerkennen. Es sei denn, man bemerkt, dass diese erstaunliche Erfolge haben und schiebt ihnen dann, das ist der Klassiker, die besonders schwierigen PatientInnen gerne $\mathrm{zu}$, ohne sie aber entsprechend anzuerkennen. Wenn in Institutionen entsprechend traumatherapeutisch ausgebildetes Fachpersonal gesucht wird, neigt man dazu 
die KollegInnen zu überfluten mit den schwierigsten PatientInnen und es gibt immer die Schwierigkeit, die Arbeit in Helfernetzwerken, zeitlich, energetisch und finanziell unterstützt zu bekommen.

JOHANNA SCHWETZ-WÜRTH: Das bedeutet, dass es dann einige wenige erfahrene und versierte Profis gibt, die, ständig überlastet, Gefahr laufen ins Burnout zu kippen?

MICHAELA HUBER: Die Befragung der Phönix-Initiative unter Fachleuten hat ergeben, dass diejenigen die mit Komplextrauma arbeiten, mehr arbeiten als andere, besser ausgebildet sind als andere, weniger verdienen und die schwierigsten KlientInnen haben! Es ergibt sich ein deutliches Bild: sobald man gut ausgebildet ist, wird man tendenziell hoffnungslos überlastet. Das muss uns ein Warnsignal sein, diese kostbaren KollegInnen gut zu unterstützten, die brauchen ganz besonders viel an Anerkennung, die müssen Zeit bekommen und dafür mehr finanziell honoriert werden: z. B. für die vielen Bescheinigungen, die sie ausfüllen, die vielen Telefonate, die sie führen müssen, um die PatientInnen zu unterstützen, die vielen „Runder-Tisch“-Gespräche, die sie führen müssen, damit sie einigermaßen dem Drama gerecht werden können. Ihre hilfreichen und oft unerlässlichen Arbeiten müssen in die Hilfeplanung einberechnet und honoriert werden. Hier gibt es ein großes Manko und das macht uns auch in den Fachgesellschaften Sorgen, weil wir sehen, dass die Ausbrenngefahr bei diesen KollegInnen so hoch ist.

Die Unterstützung von Menschen mit komplexen Traumafolgestörungen bedarf eines ganzheitlichen Zugangs und einer ganzheitlichen Finanzierung

JOHANNA SCHWETZ-WÜRTH: Das heißt, das ist ein Appell an die Institutionen, aber auch an die Krankenkassen?

MICHAELA HUBER: Bei den Kassen und dem Sozialsystem besteht das Problem, dass wir immer einzelne Therapiestunden oder Sequenzen finanziert bekommen. Es gibt kaum oder gar keine Finanzmodelle für die Finanzierung von Ausstieg aus destruktiven Bindungen, die Unterbringung von Gefährdeten, die Betreuung und Psychotherapie von Heranwachsenden und jungen traumatisierten Erwachsenen um nur einige besonders brisante Beispiele $\mathrm{zu}$ nennen. Die Ausnahme ist die Jugendhilfe, wo es manchmal Töpfe gibt, so dass verschiedene Maßnahmen aus einer Hand bezahlt werden. Das fordern wir grundsätzlich sowohl für die traumatisierten Kinder und Jugendlichen als auch für die Erwachsenen. Denn diese Menschen haben in der Regel auch irgendwann mal Kinder, sie haben Partner, sie sind mit ihrer Herkunftsfamilie verwickelt. Da haben wir multidimensionale
Problemstrukturen, Flüchtlinge sind nochmal ein Extrathema. Wir können sagen, das Problem ist die individuelle Krankheitszuschreibung plus die individuelle Finanzierung einzelner Maßnahmen ohne Zusammenschau und ohne Koordination der Hilfen - auch finanziell.

JOHANNA SCHWETZ-WÜRTH: Das ist in Österreich mindestens so schwierig ...

MICHAELA HUBER: Gesellschaftspolitisch ist das eine Fehlkalkulation. Diese KlientInnen/PatientInnen verursachen enorme Kosten. Nur als Beispiel das Thema Asthma: $20 \%$ der Asthmapatienten verursachen $80 \%$ der Kosten und zwar diejenigen, die komplex traumatisiert sind! Das ist in anderen Problemfeldern genauso - überall da wo symptomorientiert hingeschaut wird. Die komplextraumatisierten KlientInnen, also jene mit den besonders frühen bzw. besonders schweren und häufigen seelischen Belastungen, verursachen die größten Kosten im Gesundheits- und auch im Sozialsystem. Der Grund ist, weil sie weniger lang und qualifiziert arbeiten können, früher berentet werden müssen, etc. Das heißt, das ist eine Klientel, die immer spezielle Hilfen benötigt, um überhaupt weniger Kosten zu verursachen. Also auch vom reinen Kostenprinzip her müssen wir die Finanzierung der Hilfen besser gestalten. Das sehen inzwischen auch viele Organisationen und Institutionen ein. Wir werden hier sehr dicke Bretter bohren müssen und in jenen Gremien, in denen die Fachgesellschaften gehört werden, viel tun müssen.

Praxis der Traumatherapie: Vom Vertrauensaufbau zur "Gruppentherapie" mit einer einzelnen Patientin

JOHANNA SCHWETZ-WÜRTH: Ich würde gerne gegen Ende unseres Gespräches nochmal auf die Praxis der Traumatherapie zu sprechen kommen und mit dir über die größten Schwierigkeiten und Stolperfallen sprechen, denen sich auch gut ausgebildete KollegInnen, die langfristig mit komplex traumatisierten KlientInnen arbeiten, gegenüber sehen.

MICHAELA HUBER: Die größte Schwierigkeit ist erstens das Vertrauen dieser Menschen zu gewinnen. Das dauert meistens schon ein ganzes geschlagenes Jahr. Das ist wie Magie, dass meistens erst 12 Monate vergangen sein müssen und im 13. packen diese Menschen uns die schwierigsten Themen überhaupt erst auf den Tisch, weil sie uns so lange testen, weil sie so viele Bindungs- und Beziehungsstörungen haben. Zu Recht! Sie sind häufig verraten und schlecht behandelt worden, also brauchen sie lange Zeit, um Vertrauen zu fassen. Dann haben wir alle Hände voll zu tun, zu erkennen, wie die Diagnostik überhaupt vernünftig gemacht werden kann. Traumadiagnostik ist Prozessdiagnostik, das heißt erst im Lauf der 
Zeit und des vertrauensvollen miteinander Arbeitens öffnen sich die PatientInnen genügend, so dass wir erkennen, wie es um die strukturelle Dissoziation der jeweiligen Persönlichkeit bestellt ist. Hat sie ein wirklich funktionstüchtiges Alltags-Ich oder wird sie alle Nase lang von anderen Zuständen oder Innenanteilen ausgehebelt, was die Alltagsfunktionen angeht. Das erfahren wir erst im Lauf der Zeit, weil die Leute sich wahnsinnig schämen, Schuldgefühle haben und misstrauisch sind. Wenn wir dann in der Diagnostik etwas fortgeschrittener sind, dann brauchen wir Fachkenntnisse über die Arbeit auf der inneren Bühne. Das heißt, wie helfen wir als Mediatorin des Innenlebens, den unterschiedlichen Bereichen der Persönlichkeit von trauma-nahen Zuständen über das Alltags-Ich bis hin zu Täterintrojekten, sich untereinander zu verständigen, Toleranz zu bilden für Frustration und Stress, um alltägliches Funktionieren überhaupt zu ermöglichen oder aufrecht zu erhalten. Im dritten Gang müssen wir die Traumata verarbeiten helfen. Das heißt, TherapeutInnen werden bereits nach kurzer Zeit überflutet mit Erzählungen von Traumamaterial. Sie müssen diesen Prozess stark einbremsen, aber ohne dass uns die Klientin wegbricht, weil sie das Gefühl hat, wir wollen das nicht hören. Das heißt, wir müssen helfen innere Containments zu bilden, Stichwort „Sicherer Ort“, „Tresor-Übung“, etc. Es geht um Arbeit an der Binnenstruktur der Persönlichkeit, damit Schritt für Schritt die Traumatisierungen sorgsam bearbeitet werden können. Wenn die Menschen stärker dissoziieren, bis hin zu einer dissoziativen Identitätsstörung, dann haben wir es ja auch mit unterschiedlichen innen „Leuten“ zu tun (Anm: innere Teilpersönlichkeiten bei DIS), die eigene Egos haben, eigene Interessen, die sie gegen andere innere „Leute“ durchsetzen wollen, aber der Körper hat nun mal nur $24 \mathrm{~h}$. Also werden wir da eine Art „Gruppentherapie“ mit einer Einzelperson machen müssen. Und last not least werden wir große finanzielle Probleme bekommen, denn die Klientin braucht neben den üblichen therapeutischen Stunden mehr Zeit. Sie braucht Doppelstunden, Zusatztermine in Form von Telefonaten, der Möglichkeit zwischendurch zu schreiben in Form von Mails oder WhatsApp, um die Beziehung zu halten, die Einbrüche aufzufangen, etc. Dadurch bekommen wir zeitliche und eventuell auch finanzielle Probleme. Ohne Unterstützung von außen, wie etwa einer Beratungsstelle, ÄrztInnen, usw., mit denen wir zusammenarbeiten, kommen wir gar nicht aus.

\section{Nicht zu sehr einfühlen und ein gutes Netzwerk} aufbauen

JOHANNA SCHWETZ-WÜRTH: Du hast vorher schon die intensiven und in der Praxis sehr herausfordernden Übertragungs- und Gegenübertragungsphänomene angesprochen. Es gibt viele KollegInnen, die diesbezüglich in ihren Grundausbildungen nicht aus- gebildet sind. Was rätst $\mathrm{Du}$ traumatherapeutischen KollegInnen, um möglichst gut mit diesen intensiven Phänomenen umgehen zu lernen?

MICHAELA HUBER: Die vielleicht wichtigste Herausforderung ist: Versuchen Sie nie, sich in die Position der traumatisierten Klientin zu versetzen, wenn es um die Schilderungen der Traumatisierungen geht. Wenn Sie versuchen, sich so einzufühlen, als wären Sie das, der das passiert ist, brechen sie nach kurzer Zeit selbst zusammen. Denn die Klientin kann dissoziieren, Sie nicht! Die zweite Herausforderung ist, alles was die Klientin sagt, als „cum grano salis“ zu nehmen, das ist das, was diese Klientin oder der Teil der Klientin jetzt in diesem Moment wirklich denkt oder fühlt. Es ist aber nie das Ganze. Suchen Sie also nie nach dem „Eigentlichen“, denn dann werden sie sehr verwirrt. Die Klientin wird sich nämlich häufig widersprechen, viele Informationen werden disparat nebeneinander stehen, völlig unverbunden. Sie sollten wissen, dass Sie erst im Laufe der Therapie durch die Strukturarbeit der Klientin die Möglichkeit geben werden, ihre eigenen inneren Wahrheiten zu sortieren und ihre eigene Geschichte zusammen zu setzen. Eine dritte Herausforderung ist: Nehmen Sie es nie persönlich, wenn Sie attackiert werden! Sie werden vom Alltags-Ich angegriffen, weil Sie sich um die traumatisierten Innenanteile kümmern. Sie werden von den traumatisierten Innenanteilen angegriffen, weil sie sich um die Alltagsstruktur und das Funktionieren im Alltag bemühen. Und Sie werden von den Täterintrojekten angegriffen, weil Sie mit ihnen sozusagen in Konkurrenz gehen, weil Sie der Persönlichkeit in eine andere Richtung verhelfen, wie sie von den Täterintrojekten bislang propagiert wurden. Es wird länger dauern, bis auch die täterimmitierenden und täterloyalen Bereiche der Persönlichkeit tolerant und kooperativ mit Ihnen umgehen und gemerkt haben, dass Sie nicht die Konkurrenz sind.

Der große Wunsch: Trauma-Sensibilität in Medien, Exekutive, Hilfsorganisationen, Politik und Gesundheitswesen

JOHANNA SCHWETZ-WÜRTH: Welche Wünsche hast Du an KollegInnen, Versicherungen, Politik, Medien in Bezug auf den Umgang mit Traumata? Was wäre dir wichtig?

MICHAELA HUBER: Ich wünsche mir, dass die Medien nicht so invasiv die Opfer befragen, weil sie sonst Flashbacks auslösen. Da gibt es durchaus Schulungen, die man besuchen kann, etwa als JournalistIn, wo trauma-sensibel ausgebildet wird. Ich wünsche mir, dass diese Trauma-Sensibilität in allen Bereichen greift, etwa was den Umgang der Rettungskräfte mit Menschen angeht, die seelische oder körperliche Erschütterungen erlebt haben; und auch was die Polizei angeht, dass sie bei Befragungen auf jeden Fall eine 
ruhige Situation herstellen und nicht mitten im Inferno fragen. Dass die HelferInnen, die sich um längerfristige Hilfen bemühen, adäquat unterstützt werden, denn da reißt nach einer akuten Situation meistens die Hilfe schon ab. Es gibt sehr viel im Akutbereich, es gibt fast nichts für die längerfristigen Probleme und das sind jene, die die gravierendsten Schäden individuell wie gesellschaftlich und sozial verursachen. Da muss mehr Verstand hin, da muss mehr Kooperation hin, da muss solide Finanzierung und Zusammenarbeit her. Das sind vielleicht die allerwichtigsten Dinge. Und den Trauma-TherapeutInnen möchte ich einfach sagen, Sie sind so ein kostbarer Mensch, nicht nur für sich selbst, sondern auch für so viele andere Menschen. Gehen Sie sorgfältig mit Ihren Kräften um, achten Sie darauf, dass Sie selbst nicht ausbrennen, versuchen Sie früh zu erkennen, wenn Sie selbst zu viel Stress haben, damit Sie gegenregulieren. Arbei- ten Sie immer in Netzwerken von FreundInnen und HelferInnen und sorgen Sie dafür, dass Sie nicht vereinsamen - das ist vielleicht die größte Gefahr für uns alle, nicht nur für unsere PatientInnen.

JOHANNA SCHWETZ-WÜRTH: Liebe Michaela, vielen vielen Dank für das Gespräch!

Interessenkonflikt M. Huber und J. Schwetz-Würth geben an, dass kein Interessenkonflikt besteht.

Open Access Dieser Artikel wird unter der Creative Commons Namensnennung 4.0 International Lizenz (http:// creativecommons.org/licenses/by/4.0/deed.de) veröffentlicht, welche die Nutzung, Vervielfältigung, Bearbeitung, Verbreitung und Wiedergabe in jeglichem Medium und Format erlaubt, sofern Sie den/die ursprünglichen Autor(en) und die Quelle ordnungsgemäß nennen, einen Link zur Creative Commons Lizenz beifügen und angeben, ob Änderungen vorgenommen wurden. 